\title{
Téoros
}

Revue de recherche en tourisme

\section{Contribution à la science du tourisme}

\section{Jean-Michel Hoerner}

Volume 27, numéro 1, printemps 2008

Science du tourisme ou études touristiques?

URI : https://id.erudit.org/iderudit/1070891ar

DOI : https://doi.org/10.7202/1070891ar

Aller au sommaire du numéro

Éditeur(s)

Université du Québec à Montréal

ISSN

0712-8657 (imprimé)

1923-2705 (numérique)

Découvrir la revue

Citer cet article

Hoerner, J.-M. (2008). Contribution à la science du tourisme. Téoros, 27(1),

14-17. https://doi.org/10.7202/1070891ar d'utilisation que vous pouvez consulter en ligne.

https://apropos.erudit.org/fr/usagers/politique-dutilisation/ 


\section{Contribution à la science du tourisme}

\section{Jean-Michel Hoerner}

En 1995, la diffusion de plusieurs manuels techniques permet à l'Organisation mondiale du tourisme (OMT) de fixer « des lignes directrices portant sur certains problèmes complexes de définition ». L'organisation internationale est dans son rôle, et le tourisme, qui est malgré tout une activité neuve, ne peut se plaindre de son militantisme. Cependant, deux points deviennent préoccupants: I'OMT refuse d'ouvrir le débat sur ses définitions et de très nombreux spécialistes du tourisme, parfois d'éminents universitaires, lui donnent raison. Ces définitions seraient-elles donc des concepts immuables, sous prétexte qu'elles permettent une évaluation cohérente des flux touristiques internationaux? Or, il est reconnu que les concepts sont les outils propres des sciences et qu'aucun scientifique, qui fait du tourisme seulement son champ d'études, n'osera remettre en cause des définitions qu'il juge extérieures à sa discipline. C'est pourquoi nous pensons qu'il faut créer une science du tourisme pour au moins discuter de la pertinence de l'ensemble de ces définitions.

Dans notre ouvrage, Géopolitique du tourisme (2008), nous reprenons sans états d'âme les statistiques de l'OMT en matière de flux, mais, comme dans le Précis franco-anglais de tourismologie (Hoerner et Sicart, 2003), nous défendons toujours l'idée d'une science du tourisme qui propose d'autres définitions que celles de l'organisation internationale. D'ailleurs, la création de cette science n'est pas sans rapport avec le contexte mondial, dans la mesure où les activités touristiques se développent considérablement depuis la

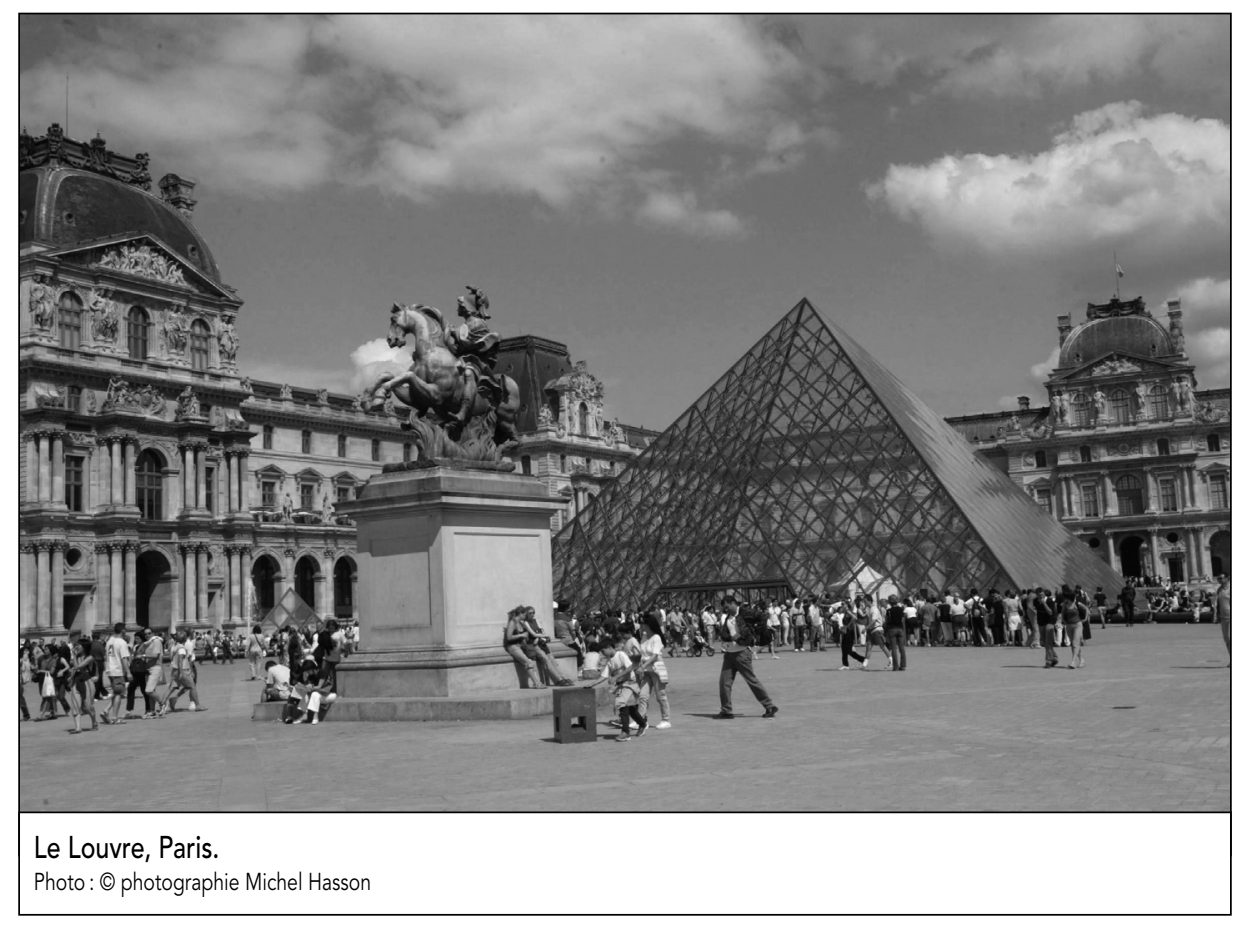

" chute des murs » de 1989-1991, c'est-àdire la fin du bloc socialiste et l'avènement de la mondialisation. D'une part, en raison sans doute d'une plus grande ouverture de toutes les frontières, les flux du tourisme international, de 1985 à 1995, se traduisent par des augmentations de $71 \%$ des entrées et de $244 \%$ des recettes. D'autre part, la mondialisation financière concerne désormais toutes les grandes entreprises touristiques, avec une multitude de fusions, d'absorptions et de spéculations boursières. Enfin, les formations supérieures en tourisme et en hôtellerie se multiplient et en France, par exemple, il y a désormais près de 20 masters en "management touristique ", tant dans les universités que dans les grandes écoles. Est-ce pour ces raisons que l'OMT exprime la volonté d'une stricte codification?

\section{La nouvelle science du tourisme et ses concepts}

Depuis l'an 2000, à la suite de plusieurs publications, nous avons lancé le débat sur l'opportunité d'une science du tourisme, qui fait partie désormais de programmes d'enseignement au Maroc, au Vietnam, en Tunisie, en Algérie, au Portugal, au Mexique, en Argentine, en Russie, en Indonésie, à Madagascar, etc. Elle est appelée « tourismologie ", bien que ce néologisme ne soit peut-être pas très heureux ${ }^{2}$. Certes, si nos convictions sont moins théoriques que pragmatiques, ce qu'on nous reproche beaucoup, il fallait bien engager le processus. Notre démarche, qui est comparable à celle de l'OMT lorsqu'elle milite pour une plus grande reconnaissance des activités touristiques, nous conduit alors à nous situer sur le terrain des formations supé- 
rieures en tourisme et en hôtellerie. Parce qu'elles sont professionnalisées, trop d'universitaires ont tendance à les sous-estimer et à les considérer comme la somme de techniques commerciales. Bien sûr, personne ne niera qu'elles aient besoin de management, d'informatique appliquée et de la pratique des langues étrangères, dont l'anglais qui est incontournable. Mais, d'une part, les techniques de management ou de marketing évoluent assez peu faute de recherche appropriée: qui se soucie, par exemple, d'améliorer la productivité dans les services touristiques et hôteliers? Personnellement, nous nous avouons incompétent pour y participer directement, mais nous savons que des spécialistes en gestion commencent sérieusement à s'y intéresser... D'autre part, et nous sommes plus à l'aise pour en parler, nous sommes conscient qu'on ne forme pas les futurs managers en tourisme et en hôtellerie sans se soucier de leur niveau. Si nous admettons qu'il s'agit de métiers de vocation - l'excessif turnover constaté nécessite des mesures -, il devient obligatoire que des formations ad hoc de type "master" s'appuient sur un corpus scientifique solide et structuré. C'est, en théorie, l'objectif de la science du tourisme.

Tout d'abord, cette science humaine et sociale à caractère pluridisciplinaire n'exclut pas les apports des sciences répertoriées dans ce domaine, et nous pouvons avancer notre propre expérience de géopolitiste ${ }^{3}$. C'est ainsi, d'ailleurs, que nous avons jugé bon de reprendre un ancien terme de la conquête française de l'Algérie au XIXe siècle, le "colonisme", qui serait lié aux séjours des touristes internationaux des classes moyennes occidentales dans les pays du Sud. Tandis que, dans leur grégarité, elles ont le comportement de colonies de vacances qui vont à l'étranger comme dans un lieu de loisir tout exprès aménagé pour elles, les populations visitées du Sud estiment qu'elles doivent se mettre au service d'étrangers seulement privilégiés par leur origine, en subissant leurs caprices, leur arrogance et, surtout, sans avoir le sentiment d'en tirer un réel profit. Nous prenons donc conscience que notre géopolitique du tourisme se situe obligatoirement dans le faisceau d'une science du tourisme qui cherche à redéfinir des concepts: le "visiteur international » n'a pas le même sens selon qu'il séjourne aux États-Unis ou au Maroc.
Cependant, nous sommes conscient que cette science du tourisme reste encore à l'état d'ébauche. Certes, des bribes de réflexion ont été avancées, et nous participons personnellement à ce débat. Mais une science, pour être vraiment reconnue, a besoin d'une intense réflexion épistémologique. En tout cas, nous nous élevons en faux contre tous ceux qui nient, à l'évidence, qu'on puisse créer une nouvelle science. II suffit de citer l'académicien Fernand Braudel qui, après avoir souligné qu'il faut tenir compte de « la prolifération des sciences nouvelles », reconnaît que si " certaines sont nées hier, d'autres naîtront demain » (2001). C'est pourquoi nous insistons sur le rôle de cette science, afin que les activités touristiques soient mieux comprises dans leur diversité et, surtout, qu'elles soient assimilées à toutes les autres activités économiques. C'est à partir de là qu'il paraît nécessaire de discuter des définitions de l'OMT ${ }^{4}$, pour lesquelles la Commission canadienne du tourisme admet volontiers que "le tourisme n'est pas une branche d'activité au sens traditionnel du terme". Sans savoir si elle le regrette, elle prétend tout de même que « le tourisme est axé sur la consommation selon le statut du client » (OMT, 1995).

Tous les chercheurs, y compris nousmême, reprenaient les définitions de l'OMT sans les discuter. Mutatis mutantis, cela ressemblait à la démarche d'économistes sans aucun sens critique, qui auraient admis sans broncher le vieil adage libéral selon lequel le profit est le seul moteur de l'économie... Toutefois, à partir du moment où certains revendiquent la nécessité d'une science du tourisme, il est logique que ses créateurs s'interrogent sur des définitions qui prennent la forme de concepts. Chacun sait que toutes les sciences sont jalouses des concepts qui les fondent, à tel point qu'on ne s'attaque jamais à ceux d'autres sciences que la sienne. Autrement dit, nous ne cherchons pas à embarrasser les responsables de l'OMT, dont nous comprenons les motivations, mais nous désirons redéfinir des concepts, voire les faire évoluer si nous le jugeons utile.

II n'en reste pas moins que la notion de concept est ambiguë, mais nous partageons la définition des géographes (Brunet et al., 1992) selon laquelle elle serait une "représentation générale, de nature abstraite, clairement définie et même consen- suelle, susceptible de guider la recherche et de fonder ses hypothèses ", en ajoutant même que "l'avancement des sciences tend vers la précision et l'enrichissement de la définition des concepts [et que] les définitions des concepts peuvent changer quand les sciences avancent ». En fait, toutes les sciences n'auraient pas les mêmes rapports avec les concepts. Certains opposent la rigueur des "notions de base" en mathématiques (Morfaux, 1980) au flou évolutif des concepts des sciences humaines... Sans vouloir faire une synthèse de manière trop systématique, nous concevons donc la science du tourisme sur la base d'Étienne Goblot: "Une science est un système de connaissances méthodiquement liées et se rapportant au même sujet. " (cité par Morfaux, 1980) Naturellement, nous souscrivons au souhait de Claude Origet du Cluzeau (2000), experte consultante, lorsqu'elle recommande «l'adoption d'un langage commun [qui] pourrait constituer une étape définitive » de la tourismologie ${ }^{5}$.

\section{Les définitions-concepts du tourisme selon I'OMT}

Deux définitions de l'OMT (1995) retiennent déjà notre attention. Elles portent sur les notions de «visiteur » et de «touriste » et ont pour but de fixer un cadre aux statistiques touristiques. Cependant, pour faciliter des évaluations mondiales, elles sont plus déterminantes qu'il n'y paraît. Tout d'abord, «le visiteur [est] toute personne qui se déplace vers un lieu situé en dehors de son environnement habituel pour une durée inférieure à 12 mois et dont le motif principal de la visite est autre que celui d'exercer une activité rémunérée dans le lieu visité ". Les visiteurs sont également des «voyageurs". Ensuite, un «touriste» est souvent « un visiteur international qui passe au moins une nuit dans un moyen d'hébergement collectif ou privé dans le pays visité »; si le visiteur est « interne », le pays visité devient un «lieu ». Quatre pleines pages explicitent ces définitions et éveillent donc notre sens critique, dans la mesure où l'OMT exclut un très grand nombre de touristes potentiels.

Dans sa conception du « voyageur », l'OMT rappelle "trois critères fondamentaux " qu'il faut détailler. Le premier exclut du champ des visiteurs tout « environnement habituel ». Si cela est logique à l'interna- 
tional, sauf pour les exceptions frontalières, cette exclusion supprime le tourisme de proximité qui, par exemple, dans le département de l'Hérault (arc méditerranéen français), contribue à plus du tiers des recettes touristiques. Par ailleurs, la limite des " 12 mois consécutifs » (autrefois, on évoquait les "vacances » qui ne devaient pas dépasser quatre mois consécutifs) met hors course les résidences hôtelières seniors, de plus en plus nombreuses, qui accueillent des retraités. N'existerait-il pas des touristes de très longue durée, dès lors qu'ils fréquentent des établissements dûment répertoriés dans la comptabilité touristique? Enfin, s'il paraît évident d'éliminer les "mouvements migratoires ", que penser des touristes d'affaires des firmes multinationales qui séjournent quelques jours dans une filiale de leur groupe? Leur salaire dépend certainement de l'ensemble des entreprises, de leur firme...

Pour le reste, les considérations relatives aux types de voyageurs dont le motif n'est pas, à l'évidence, touristique, nous paraissent assez justes : les diplomates, les forces armées, les personnes en transit (avec des nuances), etc. En matière de «tourisme interne », toutefois, on peut discuter la pertinence de ne pas prendre en compte «les personnes qui se déplacent régulièrement ou fréquemment entre des localités voisines pour des raisons de travail ou d'études ". L'homme d'affaires de Normandie qui se rend très souvent à Paris, toute proche, n'est-il pas un touriste d'affaires? Enfin, la définition du touriste par l'OMT soulève cette autre objection. En effet, s'il doit «passer la nuit », il ne peut, en aucun cas, être "un visiteur à la journée ", nommé jadis excursionniste non sans une certaine équivoque. Dans le département des Pyrénées-Orientales (sud du Languedoc-Roussillon français), la Direction départementale du tourisme recense autant les 4 millions de touristes qui passent au moins une nuit dans la région que les 4 autres millions de visiteurs qui n'y séjournent jamais plus de 24 heures. Facile à comprendre, elle ne désire pas se priver des recettes touristiques...

Après tout, c'est le droit de l'OMT de fixer ses normes dans une cohérence statistique... Faut-il pourtant faire fi des recettes touristiques et se mettre en contradiction avec la plupart des comptabilités nationales ? Dans celle de la France, où l'on dis- tingue quatre niveaux d'agrégats, au «T3 », le tout dernier, on prend ainsi en compte la "consommation touristique et para-touristique ", qui ajoute aux autres agrégats les " dépenses des visiteurs à la journée et de la clientèle locale auprès des activités caractéristiques du tourisme». Ce n'est certainement pas le «para » accolé à « touristique » qui crée la confusion : la parahôtellerie ne fait-elle pas partie de l'industrie hôtelière? On peut exclure des statistiques touristiques la clientèle locale des restaurants et les transfrontaliers qui passent une journée hors de leur pays, mais on ne peut pas rayer d'un coup de plume les recettes obtenues.

Certes, I'OMT donne des statistiques financières sur le tourisme, notamment international, mais elle ne les privilégie pas. Si cela laisse peut-être supposer que de telles données manquent de fiabilité, on pourrait également rétorquer que l'évaluation des flux est souvent sujette à caution. Dans l'espace européen, la libre circulation des personnes liée à la convention de Schengen ne favorise pas plus la comptabilité des touristes, que la mise en place de l'euro celle des recettes touristiques. II faut rattacher cette démarche particulière de l'OMT à ses définitions du tourisme. Tout d'abord, nous ne lui ferons pas un mauvais procès, dans la mesure où l'organisation internationale est soucieuse du rôle des revenus touristiques au sein des différents pays, notamment des plus pauvres. Cependant, partout, ils correspondent seulement aux dépenses des touristes et ils sont rarement perçus comme les chiffres d'affaires et les profits des entreprises, ce qui explique la priorité donnée aux enquêtes auprès des personnes. Par exemple, pour les «établissements d'hébergement », la méthode utilisée vise soit à «rassembler les données complètes dans le cadre d'entretiens avec les clients sélectionnés ", soit à « remettre un questionnaire aux clients sélectionnés pour qu'ils le complètent et le renvoient après la fin de leur voyage »...

Nous préconisons un autre choix. La démarche de la science du tourisme est plus globale et peut-être colle-t-elle aussi davantage aux réalités économiques du tourisme. Par rapport à l'OMT, elle se situerait en aval, ce qui oblige à adopter des concepts différents...

\section{Un exemple de concept pour la nouvelle science du tourisme}

Nous n'avons ni la prétention ni l'ambition de reprendre, article après article, les définitions de l'OMT, bien que ce soit sans doute un objectif très louable. Nous nous sommes pourtant attelé à une définition basique des touristes, au terme d'une réflexion assez longue et souvent contradictoire. Nous la retranscrivons in extenso (Hoerner, 2008) :

1. On fait un acte touristique lorsqu'on fait une dépense touristique, mais on devient touriste dans la durée, c'est-à-dire rarement moins d'une journée toute entière.

2. Une dépense touristique résulte soit de la nature touristique de l'établissement concerné, soit d'un séjour, même court, hors de son environnement habituel.

3. Dans ces conditions, on fait plusieurs actes touristiques, soit directement auprès des établissements ad hoc, soit dans le cadre d'un déplacement touristique qui implique autant les commerces liés au tourisme que le séjour dans une résidence secondaire, voire chez des parents ou des amis.

4. La consommation de produits touristiques, qui transforme les clients en touristes, est alors liée soit à des dépenses auprès d'établissements touristiques, soit à des séjours qui aboutissent à des dépenses touristiques indirectes, soit aux deux à la fois.

Les touristes sont donc associés, de près ou de loin, à l'industrie touristique, c'est-à-dire à une activité marchande lato sensu répertoriée dans la comptabilité nationale.

L'évaluation des flux de touristes internationaux par l'OMT n'est pas remise en cause par cette définition, car on peut admettre qu'il s'agit de visiteurs passant au moins une nuit hors de leurs pays d'origine. On regrettera cependant la non-prise en compte des visiteurs à la journée, appelés jadis excursionnistes, alors qu'on recense les touristes en transit (une nuit seulement passée dans un pays).

Cela explique les différents rapports recettes / arrivées du tourisme international selon l'OMT: moins de $400 \$$ pour la France, à fort transit, mais $625 \$$ pour l'Espagne et $2100 \$$ pour les États-Unis, où la durée des séjours est plus importante. La moyenne des 13 premiers pays touristiques 
du monde étant de 780 \$, on mesure la pertinence de cette définition des touristes.

Cette démarche explique le rôle assigné à cette science du tourisme. Nous approuvons la méthode statistique de l'OMT, mais nous donnons un second sens au terme de " touriste " dans le cadre de l'industrie touristique. II n'y a là pas plus de contradiction que dans la manière dont certains auteurs lui opposent la notion de "voyageur", qui ne serait pas toujours un simple «visiteur ». II ne faut pas circonscrire systématiquement le phénomène touristique à des considérations simplistes. Depuis que nous cherchons à élargir le débat, au sein d'une science du tourisme peut-être encore mal définie, nous avons ainsi multiplié les réflexions (nous ne sommes heureusement pas le seul) sur des thèmes beaucoup plus ambitieux. Par exemple, nous nous penchons sur le rôle du " choc des civilisations " (Huntington, 1997) dans l'invasion des touristes du Nord dans le Sud musulman (les colonistes), avec toutes ses conséquences, c'est-à-dire une certaine résistance de la dernière des grandes idéologies, l'islam, et ses dérives terroristes (Al-Qaïda). Nous ne négligeons pas non plus l'intégration de l'industrie touristique dans la mondialisation financière : rôle des fonds internationaux dans la mutation des entreprises, jusqu'à l'opération de LBO ${ }^{6}$ de Blackstone Group vis-à-vis de Hilton) ; importance de la vente des «murs » des châ̂nes hôtelières dans le but d'une recapitalisation favorable aux actionnaires; etc.

Or, sur tous ces points et sur bien d'autres encore, tel le tourisme durable, nous considérons toujours le tourisme comme une activité de marché assez classique (hormis ses « délocalisations » concomitantes d'entreprises et de clientèles). Certes, nous pourrions nous contenter de l'ensemble des sciences humaines et sociales existantes, telle la géopolitique, mais nous avons l'ambition de procéder de l'intérieur, en nous appuyant sur les fondements mêmes du tourisme. Malgré l'adhésion de beaucoup d'universitaires à notre démarche, nous savons, hélas, que beaucoup d'autres, notamment français, refusent une nouvelle science qui remettrait en cause le carcan bien établi des sciences humaines et sociales actuelles. Cela tient à la fois à leur corporatisme et, nous osons le prétendre, à leur attitude conservatrice...

\section{Conclusion}

Cet article n'a pas la prétention d'être exhaustif sur la science du tourisme que nous préconisons, de même que nous admettons que les définitions de l'OMT sont des concepts à élargir. Nous les acceptons dans un but statistique, mais nous les récusons sous leur forme réductrice. Nous considérons donc le tourisme comme une industrie, dans le sens de Jean Gadrey (1992) qui lui reconnaît les atouts d'une activité industrielle capitalistique et de maind'œuvre et, à ce titre, nous le traitons comme n'importe quelle activité économique soumise à la loi du marché. Pour autant, nous ne nions pas l'apport de scientifiques qui font du tourisme leur champ d'études. Notre propre démarche de géopolitiste le prouve... Par exemple, des historiens, des géographes, des économistes ou des psychologues intègrent dans leurs raisonnements les approches de la société que font les sociologues; or, cela n'empêche pas l'existence de la sociologie...

Si nous voulons comprendre totalement les véritables enjeux du tourisme de masse, lié à l'expansion des classes moyennes et donc, de plus en plus, à la mondialisation, nous prétendons qu'une science du tourisme répondra plus facilement à cette noble ambition. C'est ainsi le but de notre définition des touristes, qui ne répond plus au seul critère des «visiteurs passant une nuit ", mais s'inscrit dans un système plus cohérent. Et ce système, justement, permet des analyses profondes et resitue le tourisme dans le faisceau des sciences humaines et sociales. En effet, lorsqu'il est international, il n'est plus seulement le passe-temps secondaire d'une petite élite, il devient l'expression géopolitique des sociétés dominantes du Nord et la volonté d'émancipation des sociétés dominées du Sud. Ce serait alors l'enjeu d'une science du tourisme de permettre de situer ce phénomène de société au cœur de la problématique du monde, de manière globale et directe, sans avoir recours à toutes les autres sciences, la géographie, la géopolitique, la sociologie, etc., pour y parvenir.

Jean-Michel Hoerner, professeur de géopolitique et de tourisme, doyen de la Faculté "Sport, Tourisme, Hôtellerie Internationale » de l'Université de Perpignan Via Domitia.

\section{Notes}

1 Lorsque nous évoquons les "sciences " du tourisme, nous faisons référence à toutes les sciences qui le considèrent comme " champ d'études ». En revanche, cette science du tourisme, qui ne rejette pas les apports scientifiques des sciences déjà bien établies, se veut innovante et capable de redéfinir les concepts qui lui sont propres.

2 Certains auteurs ont proposé la " touristologie ", qui présente cependant une connotation trop axée sur les touristes, et des chercheurs américains ou canadiens lui préfèrent la « tourologie » ou la «téorologie ». En privilégiant « la science du tourisme", nous évitons sans doute les vaines querelles de sémantique...

3 Nous venons de publier aux Éditions Ellipses (Paris, 2008), une Géopolitique du capital.

4 Nous saluons, comme il se doit, ce corpus de définitions qui demeure très complet.

5 Lors du Forum international de l'Association mondiale pour la formation hôtelière et touristique (Amforth), une première définition de la tourismologie a été adoptée: "Ses méthodes se situent à l'interférence des sciences humaines et sociales, mais développent également ses outils et ses concepts spécifiques. La finalité de la tourismologie consiste à élever le niveau des formations professionnalisées en hôtellerie et tourisme, à 'autonomiser' une recherche déjà active, à optimiser les outils de travail des professionnels du tourisme et à adosser la branche tourisme à un corpus de connaissances scientifiques .»

6 Le Leverage Buy Out ou LBO est une opération spéculative visant à racheter une entreprise en s'endettant.

\section{Bibliographie}

Braudel, Fernand (2001), Les écrits de FB, l'Histoire au quotidien, Paris, De Fallois.

Brunet, Roger, Robert Ferras et Hervé Théry (1992), Les mots de la géographie, dictionnaire critique, Paris, Reclus-La Documentation Française.

Gadrey, Jean (1992), L'économie des services, Paris, Repères / La Découverte.

Hoerner, Jean-Michel (2008), Géopolitique du tourisme, Paris, Armand Colin.

Hoerner, Jean-Michel et Catherine Sicart (2003), La science du tourisme, Précis franco-anglais de tourismologie, Baixas, Balzac Éditeur

Huntington, Samuel P. (1997), Le choc des civilisations, Paris, Odile Jacob.

Morfaux, Louis-Marie (1980), Vocabulaire de la philosophie et des sciences humaines, Paris, Armand Colin.

OMT (1995), Manuel technique $n^{\circ} 2$ : rassemblement des statistiques de la dépense touristique, Madrid, OMT. 